\title{
Article \\ D-PARK: User-Centric Smart Parking System over BLE-Beacon Based Internet of Things
}

\author{
Soochang Park
}

Citation: Park, S. D-PARK:

User-Centric Smart Parking System over BLE-Beacon Based Internet of Things. Electronics 2021, 10, 541. https://doi.org/10.3390/ electronics10050541

Academic Editor: Jemal H. Abawajy

Received: 6 January 2021

Accepted: 23 February 2021

Published: 25 February 2021

Publisher's Note: MDPI stays neutral with regard to jurisdictional claims in published maps and institutional affiliations.

Copyright: (C) 2021 by the author. Licensee MDPI, Basel, Switzerland. This article is an open access article distributed under the terms and conditions of the Creative Commons Attribution (CC BY) license (https:/ / creativecommons.org/licenses/by/ $4.0 /)$.
Department of Computer Engineering, Chungbuk National University, Cheongju 28644, Korea; cewinter@chungbuk.ac.kr; Tel.: +82-043-261-2262

\begin{abstract}
Our daily life services are quickly becoming smarter with intelligence and information through artificial intelligence (AI) and Big Data technologies. Parking services are one of the most frequently used in our daily life-cycle. This parking application could be classified into several features according to demands and properties, such as parking capacity balancing on a city-level view, parking fee maximization for achieving the service provider demand, empty parking spot notification within a parking lot, etc. This paper concentrates on parking space detection and alert to users. Most smart services rely on smart mobile derives of users such as smartphones and smartwatches. The proposed novel mechanism for smart parking is based on a smart device to gather mobile sensing data such as users' activity and position data. Acquired mobile data are analyzed via machine learning technologies to provide dedicated parking services per user. Based on real testbed setups on campus and the proof-of-concept implementation, the proposed localization can achieve accuracy of a parking spot scale ( $2 m$-second guess 95\%); moreover, it shows a much lower service operation period of 6.8 times (34s) than the legacy approach (230s).
\end{abstract}

Keywords: smart parking; Internet of Things; Bluetooth Low Energy; status sensing; proximity detection; anlaytics

\section{Introduction}

Nowadays, a wide range of smart systems are delivered as a result of the growth of mobile networks, sensor networks, and the ubiquity of computing and cloud computing [1]. Smart systems for everyday demands such as parking, transporting and working have been sustainedly developed to achieve higher system performance and efficiency [2-7]. However, such smartness for systems has led to the failure to address the experience of end users since they have missed user situation and intent to deliver smart services [8,9]. For instance, the current smart parking systems follow a steep learning curve, which requires drivers to have an average computer literacy before they get familiar with the operating environment of the smart parking application. Even this system is not well matched with users' personal demand and intent. Apparently, both the unfriendliness operation and misunderstanding of user demands have severely affected drivers' experiences both before and after parking.

There is a diverse variety of car parks present in urban environments, such as onstreet parking lots, parking buildings, and underground parking garages. In a car park, Global Positioning System (GPS) and WiFi signal strength based localization are commonly employed for parking position detection of vehicles [2-14]. GPS is usually used to indicate the position of a parking lot due to its large error scale and restriction in indoor environments [15]. On the other hand, WiFi-based technologies such as WiFi fingerprint or trilateration can offer meter-scale localization, but they are not precise and flexible enough for vehicle position detection. If a car park is an outdoor parking lot, they do not effectively meet requirements since signals are usually lower in outdoor environments. Moreover, $\mathrm{WiFi}$ is an expensive technology considering the number of access points required for a big 
parking lot or multi-layered parking building. According to a state-of-the-art research [14], the accuracy of WiFi fingerprint with one access point per $100 \mathrm{~m}^{2}$ is $8.5 \mathrm{~m}$ in an indoor place. This means that WiFi-based techniques cannot achieve the approximately two meter-scale localization required to distinguish each parking spot at indoor/outdoor locations.

Apple Inc. introduced iBeacon in 2013, and Google launched Eddystone in 2015, which are Bluetooth low energy (BLE)-based beaconing systems. The beacons of such systems are battery-functioned small devices that broadcast IDs to proximity periodically. Up-to-date research has proposed more accurate localization or indoor position-based applications $[14,16]$. Meanwhile, smart mobile devices have become popular for sensing user behaviours and actions via embedded sensors on mobile devices such as GPS, accelerometer, gyroscope and compass sensors [15-19]. This sensing can help to elucidate the user situation. However, existing studies for BLE-beacon-based localization do not report parking spot scale accuracy (i.e., to about $5 \mathrm{~m}$ [14]). Such user sensing technologies could not support the smart parking application, which has more comprehensive processes and scenarios since they have been designed for single-action scenarios to limit applying sensed and signal data.

This paper proposes a novel smart parking system to breakthrough such restrictions of previous studies in terms of positioning accuracy and user understanding, named the userDedicated PARKing system (D-PARK). This newly proposed system extends the previous work [10] that involved a smart parking mechanism with localization with static radio channel setup and simplified user detection. D-PARK consists of three layers: (1) highresolution localization with dynamic channel data as a real-world setup, (2) comprehensive user and vehicle status sensing, (3) user intent analysis relied on a concatenated denoising autoencoder to improve user detection accuracy. D-PARK is developed with BLE-beacon based IoT infrastructure, smart mobile devices with sensors and mobile applications and cloud systems. The signals of BLE beacons are analyzed and processed for high-resolution localization against different scanning intervals. All sensor and signal data derived by smart mobile devices such as GPS, beacon, WiFi, accelerometer, gyroscope, and illumination are investigated to determine their feasibility and applicability for users and vehicles' status sensing in parking scenarios. Based on such investigation and status sensing, D-PARK could provide user intent detection for accomplishing the ultimate goal of this paper, i.e., high-level user experiences in parking places. In this paper, the proof-of-concept over a real testbed at the outdoor on-street parking lot on the campus is implemented. All experiment results are conducted through real testbed and implementation. Figure 1 illustrates architectural overviews and core layers of D-PARK.

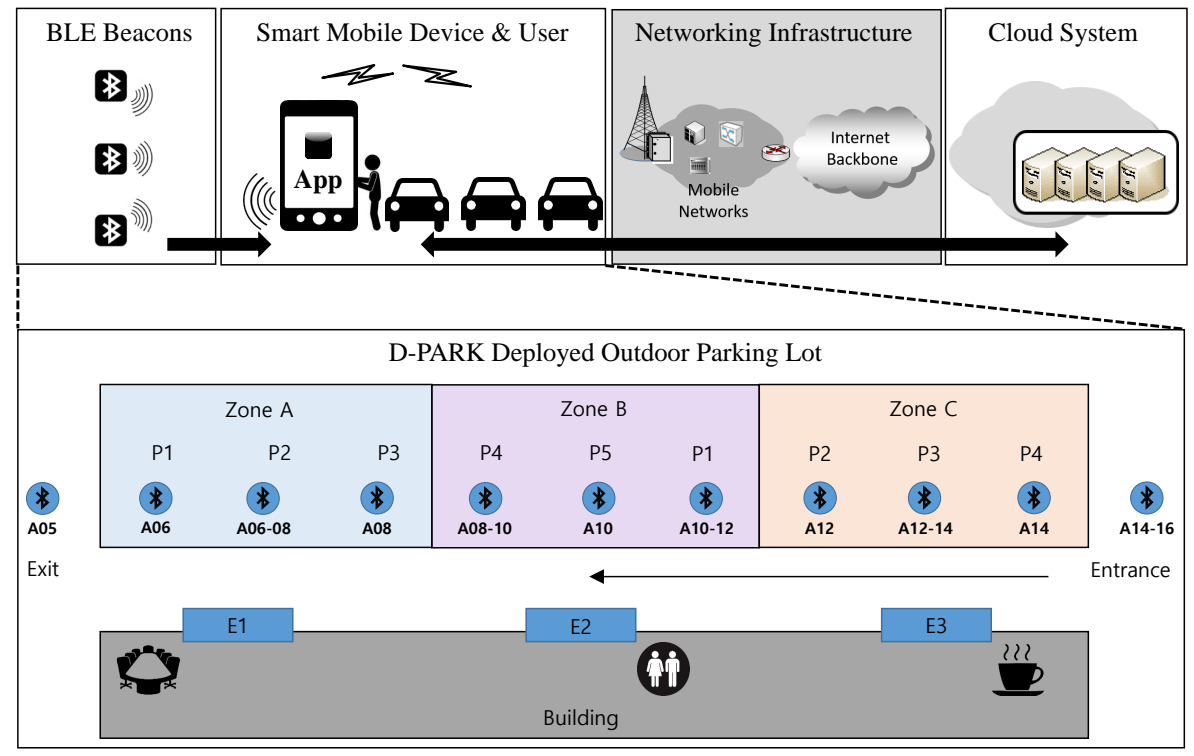

Figure 1. D-PARK architecture overview and testbed of outdoor parking lot. 
The rest of this paper is structured as follows: Section 2 explains related work; Section 3 presents high-resolution localization; Section 4 addresses user/vehicle status sensing and user intent detection with data analysis data from mobile devices and shows the results of the evaluation; and Section 5 concludes the paper and provides future work.

\section{Related Works}

The previous studies on smart parking systems have mainly dealt with available parking space search and parking fee reduction among multiple parking lots. For these purpose, the SPARK scheme [2] proposes a parking scheme for large parking lots through vehicular communication. SPARK provides the drivers with a real-time parking navigation service and friendly parking information dissemination. In addition, another system [3] is based on intelligent resource allocation, reservation, and pricing. The proposed system solves the current parking problems by offering guaranteed parking reservations with the lowest possible cost and searching time for drivers and the highest revenue and resource utilization for parking managers. The other system [4], named iPaker, is proposed for smart parking for an urban environment. iPaker assigns and reserves an optimal parking space based on the driver's cost function, which combines proximity to destination and parking cost. In [5], C. Tang et al. provides an available parking lot information service in a citywide area by the vehicular ad hoc networking (VANET) and fog computing technologies. In addition, [6] introduces the on-street parking place search technology based on sonar and a camera embedded on a vehicle that has GPS devices and cellular communication channels.

Indoor positioning is a mature research field [11-14,16]. The focus is on positioning through pattern-matching the current signal detection to a previously surveyed map of signal strengths. Today, the most common technology used in indoor places is WiFi [14]. WiFi positioning is tightly integrated into many mobile platforms, providing urban localization on the scale of about $10 \mathrm{~m}$. WiFi signal pattern matching, or fingerprinting, is the de-facto localization technique for indoor positioning on user devices today. Other candidates for radio fingerprinting are cellular and Bluetooth signals. Cellular sources are typically too sparsely distributed to provide good indoor fingerprints, and most notably, they require very lengthy scan times. The recent introduction of the Bluetooth 4.0 specification has potentially addressed these problems via Bluetooth Low Energy (BLE). The recent work for indoor BLE beacon fingerprinting [14] shows an accuracy to about $5 \mathrm{~m}$.

Recent smart interactive systems are commonly based on notification by BLE advertising to smart mobile devices such as smartphones and tablets. When a user receives a notification in terms of availability of interaction, i.e., smart mobile devices receive a BLE advertising message, the user can recognize this smart method. A smart home application is built with BLE beacons for interactive services and energy saving [16]. Interactive digital signage is exploited to increase the effectiveness of advertising [20]. For the edutainment sector such as museums, galleries, exhibition halls, and classrooms, smart mobile devices-beacon interactive features can enhance the users' learning experiences [21].

In consequence, the existing smart parking systems are mainly based on the high utilization of parking spots over multiple parking lot environments. The accuracy of locating cars is merely a car park level or parking zone scale, based on GPS or WiFi, in common. However, D-PARK provides an empty parking spot notification rather than achieving high utilization, while also obtaining the position in high-resolution by BLE rather than by GPS or WiFi.

\section{D-PARK: Dedicated Smart Parking System}

Urban public spaces are being progressively fitted with Internet of Things (IoT) devices composed of ambient sensors, actuators and beacons running Bluetooth Low Energy (BLE) that help to offer continuous observability and interactivity from/to physical environments. Each beacon device constantly broadcasts its unique identifiers to nearby smart mobile devices to perform creative interactions between a mobile application and an associated cloud system for users in close proximity. So, the clustering of such paradigms 
(i.e., BLE beacon-based IoT infrastructures, smart mobiles and cloud computing) is clearly a compelling and timely research issue. Figure 1 shows this joined architecture for smart applications. Here, the BLE beacon infrastructure is deployed at indoor place as well as outdoor place to support user positioning for parking services. This smart parking is based on the smart mobile device of users who are the drivers in a parking lot. Such user location and user status are analyzed in the cloud to provide parking spot notifications, easy checkout, and building entrance/parking place information. Figure 1 also presents the testbed of proposed parking famework, named D-PARK. Then, based on this systemic infrastructure, D-PARK is primarily operated as mobile applications and a system cloud. In mobile applications, D-PARK has three key functionalities as follows:

1. High-resolution localization through signal processing of BLE beacons with machine a learning technology with dynamic scanning interval;

2. User and vehicle status sensing via on data processing of all sensor and signal data of a smart mobile device with machine learning;

3. User intent detection based on the status sensing and inter-operation with a cloud system.

In addition, in the cloud, there are four main functionalities such as smart space environmental and deployed equipment manager, user and content managers for parking and its relevant services, and push notification manager.

\subsection{High-Resolution Location Estimation}

In order to empower a smart parking system with capabilities to make recommendation and analytics based on occupancy rate, localization of the user/vehicle plays a pivotal role. However, to enable this vision, construction of reliable and scalable infrastructure and the corresponding localization method is vital. In this section, a high-resolution proximity estimation system, a novel localization method that utilized BLE beacon infrastructure, is introduced. Commonly, BLE beacons are not suitable for fine-grained localization applications due to its severe fading effects. Consequently, BLE beacons were mainly used for applications that require proximity detection, where each beacon was mapped to a single physical location to provide contextual/locational information. However, proximity-based infrastructure is quite expensive due to the number of beacons required. The proposed system attempts to improve the existing proximity-based infrastructure, by compromising accuracy for its deployment cost.

\subsubsection{Difference of Scanning Interval}

To conduct this experiment, a testbed of the BLE beacon infrastructure was constructed, as shown in the figure. The testbed was composed of 10 BLE beacons, configured at an advertising interval of $800 \mathrm{~ms}$ and a transmission power of $0 \mathrm{dBm}$. To collect training data, the received signal strengths of BLE beacons at each of the parking spots were recorded for $1 \mathrm{~min}$. Raw data were constructed with the following elements: time of reception is $\mathrm{ms}$, mac address of BLE beacon, RSSI value, parking spot where the signal was received. Raw data consisted of 1425 training examples. Therefore, raw data are a $4 \times N$ matrix, where $N$ is the number of training examples.

Raw data are conditioned and pre-processed for the training process. Firstly, raw data are separated into feature and output matrices. Both of these matrices are converted into sparse matrices of dimension $d \times N$ and $k \times N$, respectively, where $d$ is the number of features or RSSI values of each beacon, and $k$ is the number of classes or parking spot locations. During the pre-processing stage, raw data are averaged over a given scanning interval to create a new feature matrix of the same dimensions. Once the matrices are conditioned and pre-processed, they are divided into training and validation set with a ratio of 4:1. 
Logistic regression was used to predict the current location of the user, under the assumption that the user has just parked. Softmax function was used to estimate the probability that the user was at a certain location $i$, given the feature vector, $x$ :

$$
\hat{P}\left(C_{i} \mid x\right)=\frac{\exp \left(w_{i}^{T} x+w_{i 0}\right)}{\sum_{j=1}^{i} \exp \left(w_{j}^{T} x+w_{j 0}\right)},
$$

where $C_{i}$ is the class $i, x$ is the feature vector, $w_{j}$ is the weight for $j t h$ class, and $w_{j 0}$ is the constant term for the given weight. The cross-entropy function was used as the cost function:

$$
E(w \mid x)=-\frac{1}{m} \sum_{l=1}^{m} \sum_{i=1}^{k} r_{i}^{(l)} \log \hat{P}\left(C_{i} \mid x^{(l)}\right) .
$$

To train this system, the gradient descent algorithm was employed, and the corresponding updated equation was derived for the weights based on the cross-entropy function:

$$
\begin{aligned}
& \Delta w_{j}=\eta \sum_{l}\left(r_{j}^{(l)}-y_{j}^{(l)}\right) x^{l}, \\
& \Delta w_{j 0}=\eta \sum_{l}\left(r_{j}^{(l)}-y_{j}^{(l)}\right),
\end{aligned}
$$

where $\Delta w_{j 0}$ is the update rule for the constant terms, $\Delta w_{j 0}$ is the update rule for features in training vectors, $\eta$ is the update rate, $y_{j}^{(l)}$ is the predicted class, and $r_{j}^{(l)}$ is the actual class.

To investigate the effect of the scanning interval on the accuracy of the system, the system was trained multiple times with different values of scanning interval ranging from $1000 \mathrm{~ms}$ to $30,000 \mathrm{~ms}$. Figure 2 shows the accuracy of the system tested on the validation sets 10 times. It can be seen that at scanning interval of $10 \mathrm{~s}$, the accuracy of first guess is $85 \%$, second guess, $95 \%$, and third guess, nearly $100 \%$. As expected, a positive correlation between the scanning interval and accuracy can be observed. However, it is interesting to note that these values converge at some point when the scanning interval is too long.

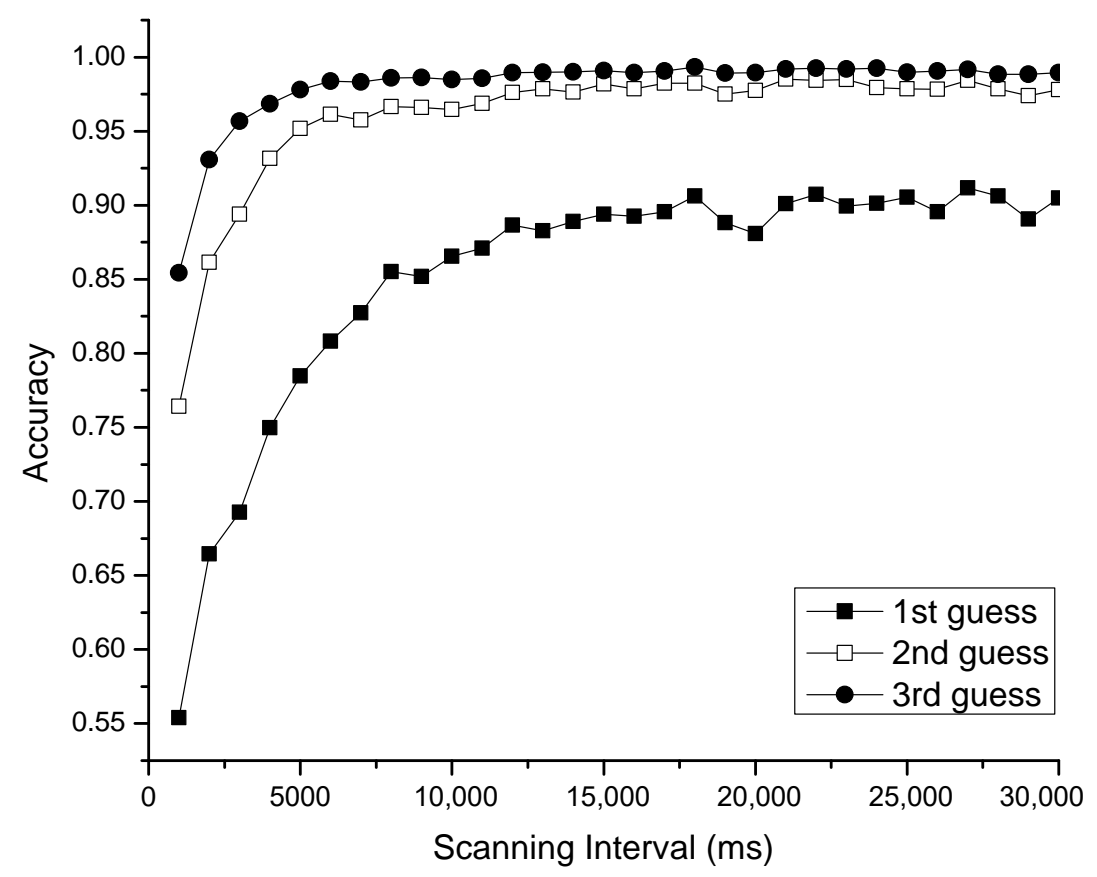

Figure 2. Accuracy of the system plotted against scanning interval.

\subsubsection{Concatenated Denoising Autoencoder}

Urban public spaces are being progressively fitted with Internet of Things (IoT) devices composed of ambient sensors, actuators and beacons running Bluetooth Low Energy (BLE) 
that help to offer continuous observability and interactivity from/to physical environments. Each beacon device constantly broadcasts its unique identifiers to nearby smart mobile devices to perform creative interactions between mobile applications and an associated cloud system for users in close proximity. So, the clustering of such paradigms (i.e., BLE beacon based IoT infrastructures, smart mobiles and cloud computing) is clearly a compelling and timely research issue.

D-PARK detects the user's location in the parking lot using a mobile device and analyzes whether the user is inside or outside the vehicle (user status). As shown in Figure 3, this system consists of three elements: BLE beacon, mobile devices, and edge server. BLE beacons send the broadcast signal and the mobile devices collect the BLE beacon signals, which are used to estimate the user's location. The edge server analyzes the user's location. The system models are detailed as follows.
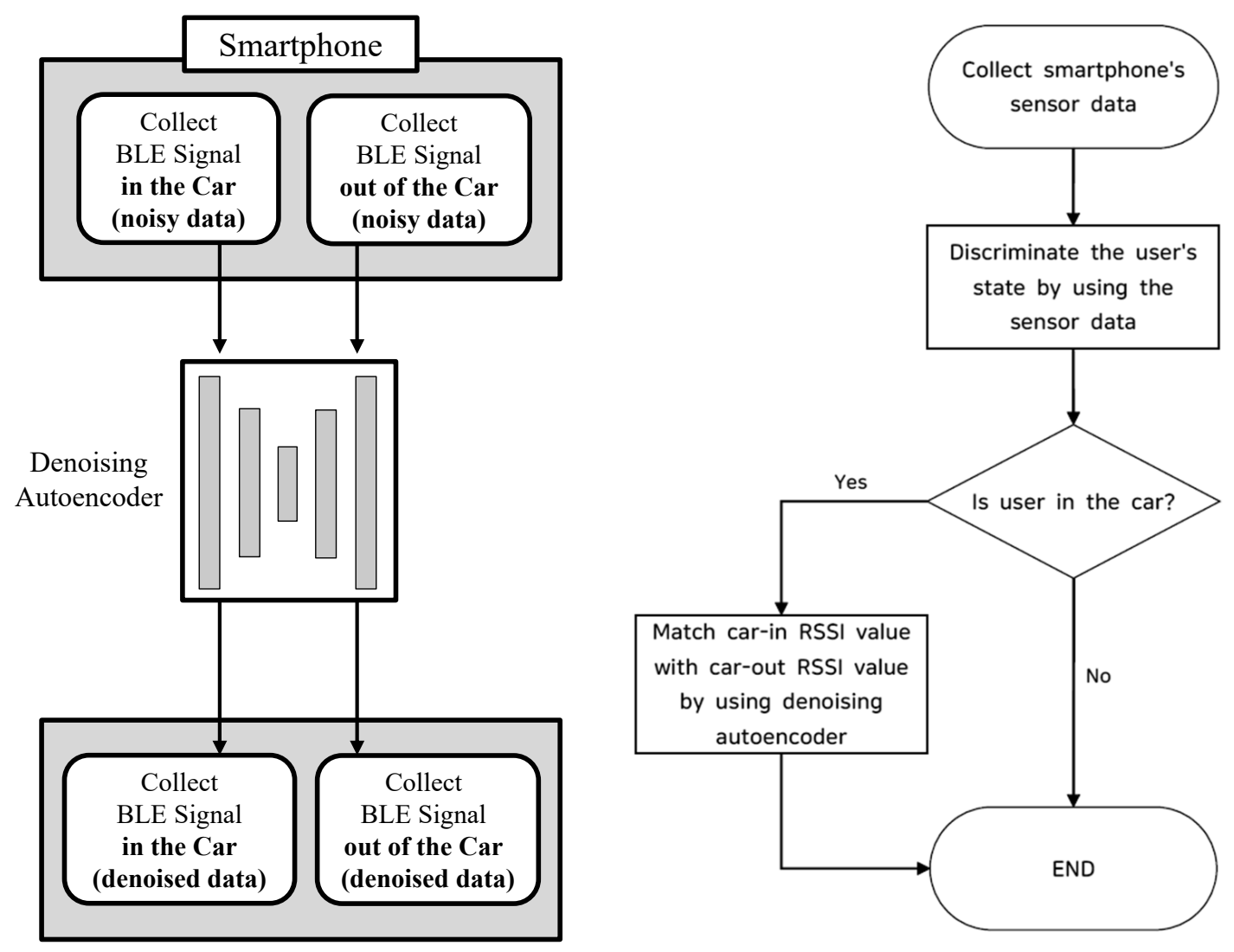

Figure 3. Concatenated Denoising Autoencoder Framework.

In mobile devices, the sensor data and radio signal strength indicators (RSSIs) of BLE beacons are collected. RSSI of BLE beacons are collected both in and out of the vehicle. The mobile device sends the sensor data and RSSIs to the edge server.

The edge server estimates the user's location and state by using sensor data and RSSIs, which are collected by the user's mobile device. In the other words, the edge server trains the deep learning model with these data. We use and compare the train Multi-layer Perceptron (MLP), 1D Convolutional Neural Network (1D-CNN), and Support Vector Machine (SVM) models [22]. The process of estimation in the edge server is as follows.

1. The raw data from inside the vehicle pass denoising autoencoder.

2. The denoised data are grouped and the averages are calculated.

3. The averaged data pass converting autoencoder and become the data with the form of the data from outside.

4. The deep learning model estimates user's location from converted data. 
The RSSI data contain a lot of noise, which obstructs the exact estimation of the user's location. We remove the noise by using a denoising auto encoder, which results in Figure 4 . The inputs of the denoising autoencoder are raw (noisy) data and the noice is thereby mitigated from the raw data. In the training process, we use the input and the result data. The input data are raw RSSIs of BLE beacon and the result data are RSSIs, which mitigated noise by using the measured value. The values are calculated as mean and standard deviation of the data with the same label. By doing so, the data that are far from the mean can be modified to make them closer to the mean.

a)

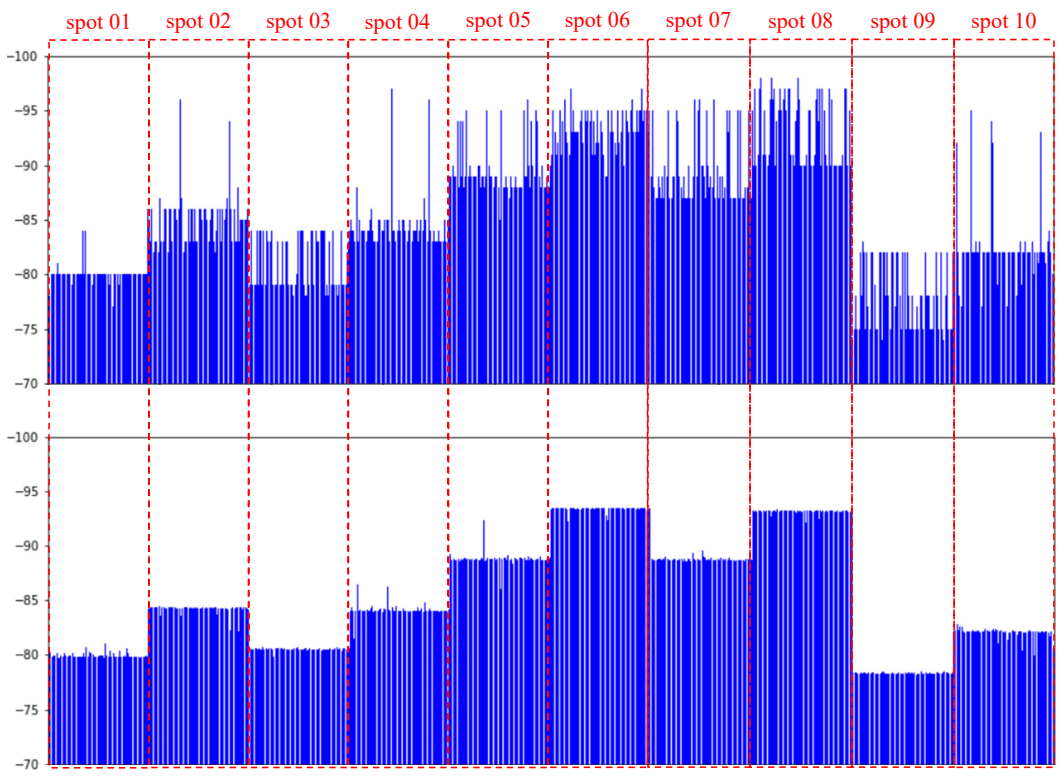

Figure 4. Denoising RSSI Graphs: (a) noised RSSI (b) RSSI after denoising.

A converting autoencoder converts the form of the grouped data from the in-car data into the out-car data in Figure 5. By doing so, the system model can estimate the user's location from using only one deep learning model regardless of the user in-car or out-car situations.

a)

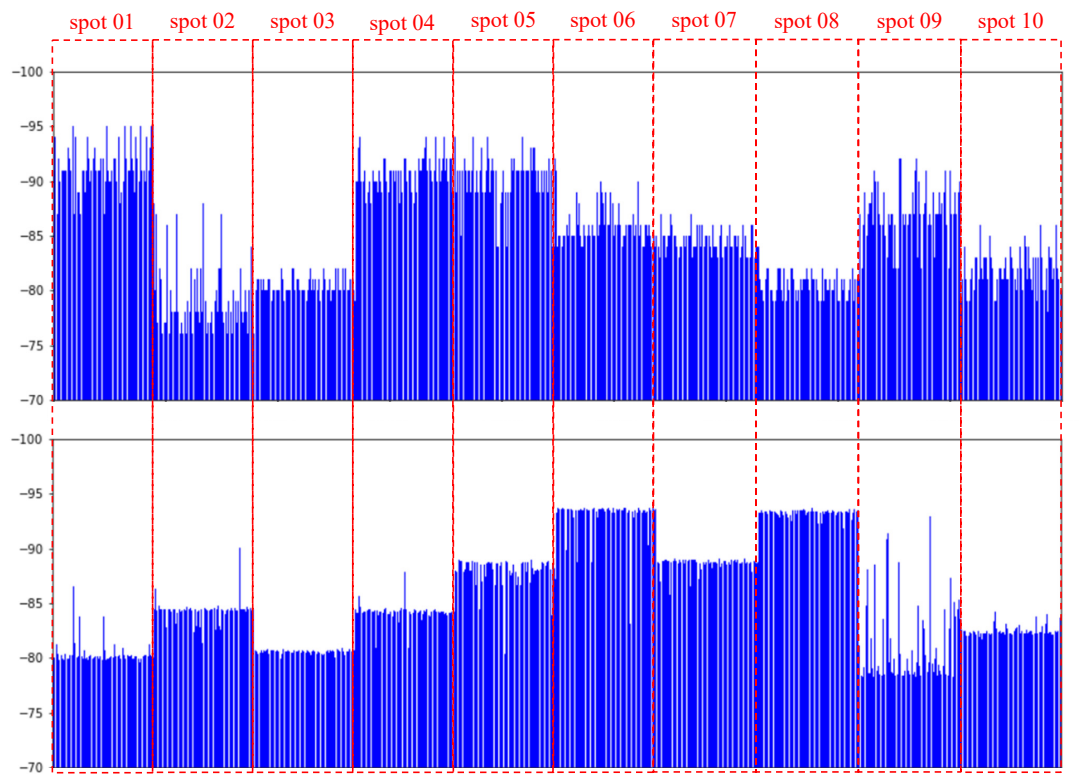

Figure 5. Converting RSSI Graphs: (a) RSSI inside a Car (b) converting to outside RSSI. 
In the proposed system, the ways of preprocessing the data are different depending on whether the data are from inside the vehicle or outside the vehicle. However, it is difficult to find out whether the user is inside or outside the vehicle by using only RSSIs of BLE beacons. That is why we use sensor data collected from a smartphone. Among the lots of sensor data from the smartphone, the magnetometer shows very meaningful data for detecting user status related to the vehicle. By training MLP, 1D-CNN, SVM model using sensing data from the magnetometer, we predict whether the user is inside or outside the vehicle. As a result, it is possible to preprocess the data appropriately for the user status.

Unlike the work in [10] which is based on separated networks for in and out of a car to detect location, SVM has $74.5 \%$ accuracy for the out-car data and $92.2 \%$ accuracy for the in-car data in the same trained model. MLP has $98.1 \%$ accuracy for the out-car data and $94.9 \%$ accuracy for the in-car data. CNN has $98 \%$ accuracy for the out-car data and $94.5 \%$ accuracy for the in-car data. The highest accuracy of out-car data is $98.1 \%$ and that of in-car data is $94.9 \%$ in the same trained model.

\subsection{Status Sensing and Intent-Based Reactions}

This subsection explains procedures of user and vehicle status sensing, which are addressed in the Table 1, through BLE beacon signals, GPS, accelerometer, magnetic sensor, and light sensor. Although many previous studies mentioned restriction of some wireless signals and sensors are not affordable and feasible for user status sensing or indoor/outdoor positioning, we have done our own preliminary experiments on all possible sensor and signal data. By preliminary experiments, we have ensured that temperature, WiFi, cellular, and pressure factors were not considered for this parking system case. On the other hand, light sensors, magnetic sensors, accelerometers and GPS show very meaningful data for detecting user and vehicle status. As shown in Figure 6, light sensor data are dramatically decreased when a user gets inside a building. However, users do not carry their smart mobile device on their person every time. So, here we also consider alternative meaningful data so that GPS is taken into consideration. Figure 6 illustrates the number of the Satellite for GPS detection from 13 to 1. In D-PARK, the criteria are chosen based on these preliminary experimental results for user and vehicle status sensing.

Table 1. Status, Intent and Reactions.

\begin{tabular}{|c|c|c|c|}
\hline Case & No. & Status and Intent & Reactions \\
\hline \multirow{5}{*}{ Come } & 1 & Approach to the parking lot & \multirow{5}{*}{$\begin{array}{l}\text { Welcome message } \\
\text { Empty/preferred parking spot recommendation } \\
\text { and navigation } \\
\text { Keeping on recommendation } \\
\text { Parked spot notification and the nearest entrance } \\
\text { recommendation according to user goals } \\
\text { 'Welcome Building' message \& direction notification to the } \\
\text { destination of user }\end{array}$} \\
\hline & 2 & Search a parking spot & \\
\hline & 3 & Waiting for parking & \\
\hline & 4 & Parked/Get off the car & \\
\hline & 5 & Get into the associated building & \\
\hline \multirow{3}{*}{ Return } & 6 & Return to the parking lot & Notifying parked spot and direction to it \\
\hline & 7 & Pay & Informing parking fee \\
\hline & 8 & Get in the car & Notifying direction to the exit \\
\hline \multirow{2}{*}{ Leave } & 9 & Leaving & Keep on notifying \\
\hline & 10 & Left & 'Good-bye' message \\
\hline
\end{tabular}



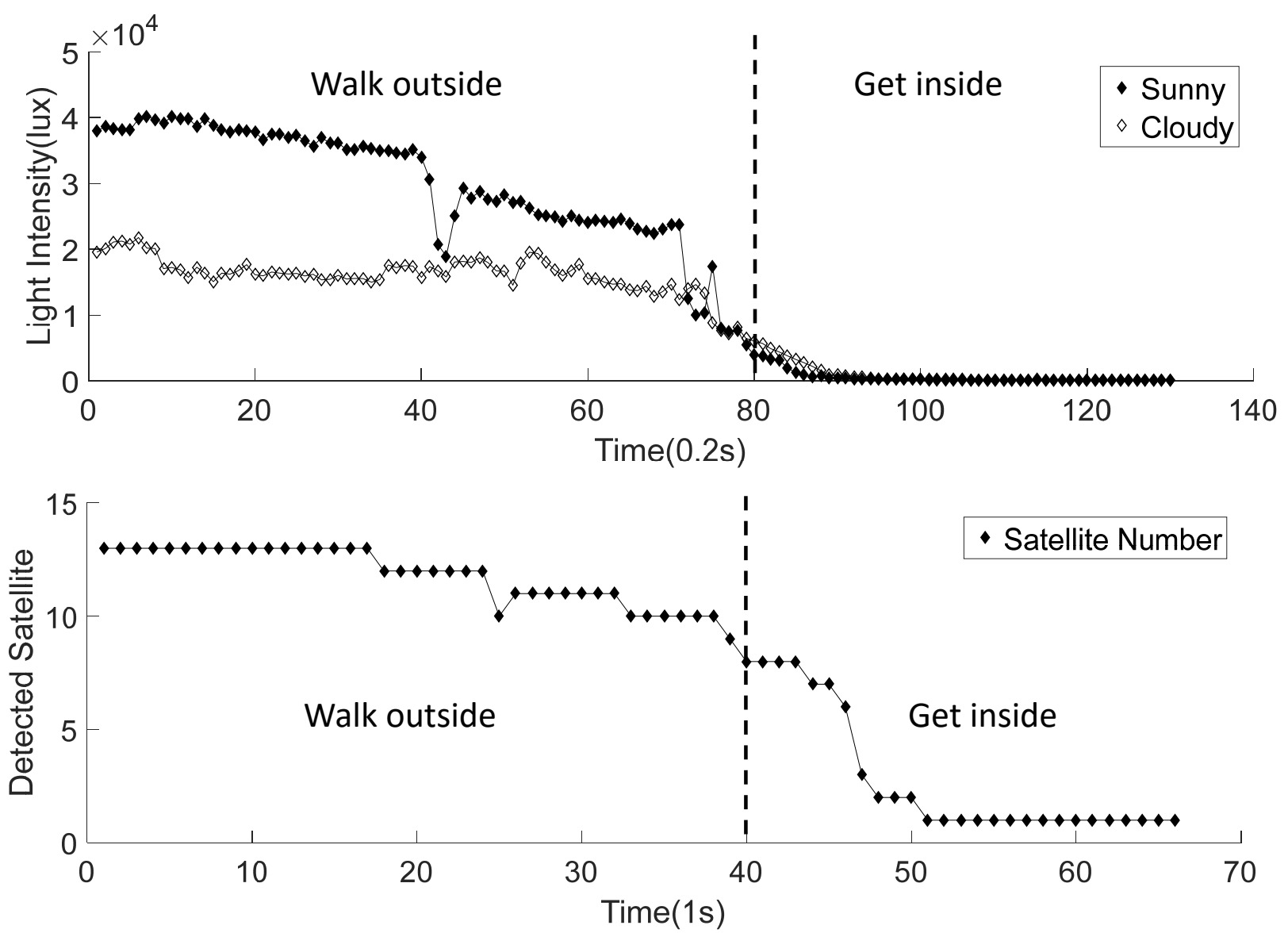

Figure 6. Illumination and GPS change between inside/outside a building.

In addition, the comparison of accelerometer and magnetic sensing data shown in Figure 7 explains interesting and meaningful results. An accelerometer is the most common sensor for behavior sensing by smart mobile devices; however, it is too sensitive. That is, the data of an accelerometer are measured highly frequently and quickly change and fluctuate, so it is not easy to differentiate user status and behavior. Hence, D-PARK considers a combination of acceleromater data with magnetic sensor data, as shown in Figure 7.

The flow chart of Figure 8 presents D-PARK operations for smart parking with localization, status sensing and intent-based reactions. Each status, intent and associated reactions are explained in Table 1 . Based on the status sensing technique such as detecting 'walking' or 'driving', user intent could be derived from joining of not only status sensing results but also BLE beacon signal status. Such comprehensive detection is able to increase the status sensing accuracy, as shown in Figures 6 and 7. Table 2 addresses user intent detection by comprehensive status sensing of users and vehicles. 

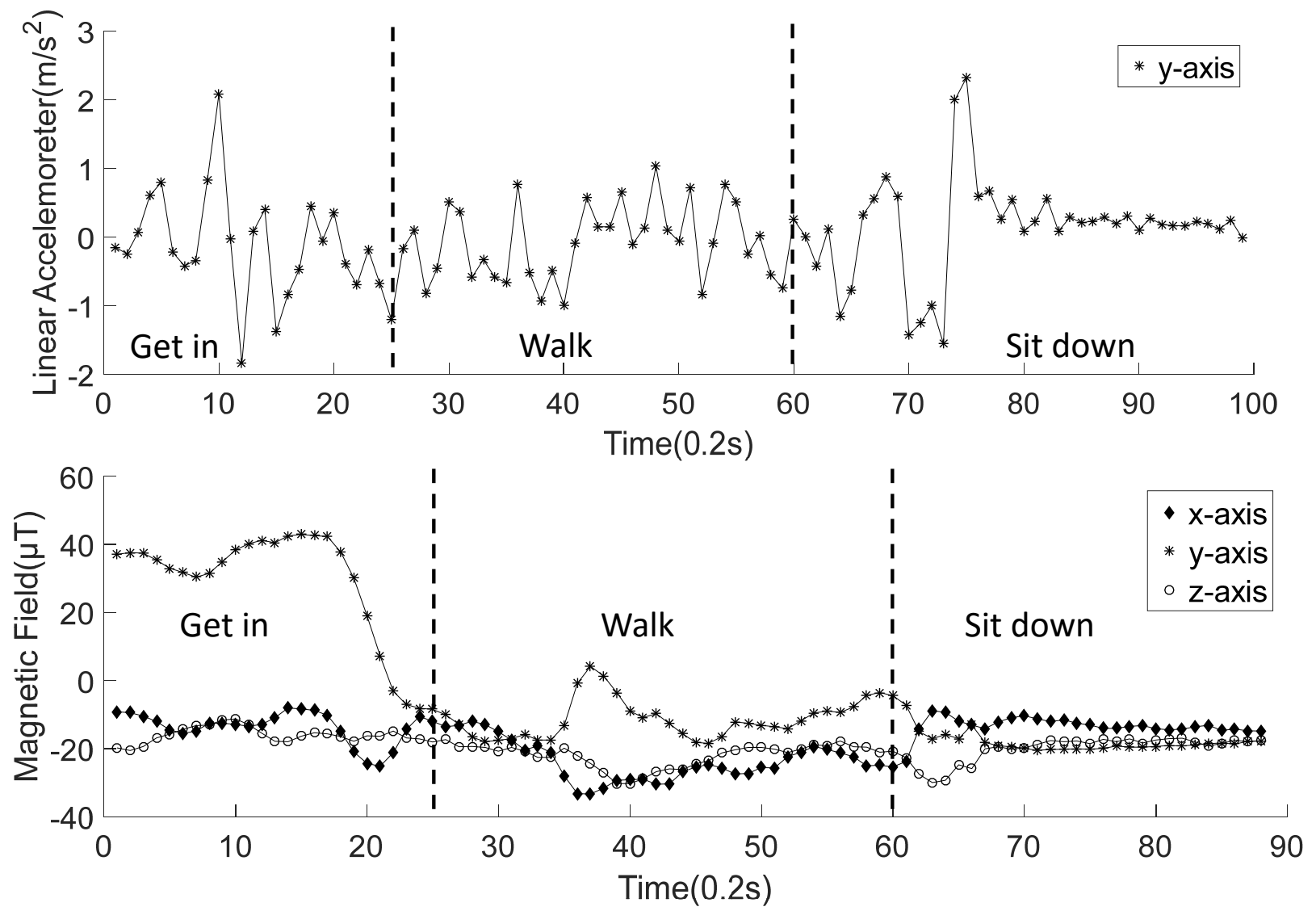

Figure 7. Accelerometer and magnetic data change in/out of a car.

Table 2. User Intent Detection.

\begin{tabular}{cc}
\hline Intent & Methodology \\
\hline Parking spot search & $\begin{array}{c}\text { After entrance beacon detection; } \\
\text { Driving status }\end{array}$ \\
\hline Waiting for parking & $\begin{array}{c}\text { Driving, then Stop; } \\
\text { Same beacons detection }\end{array}$ \\
\hline Building entrance search & $\begin{array}{c}\text { Parked; } \\
\text { Walking }\end{array}$ \\
\hline Parked spot search & $\begin{array}{c}\text { Getting outside; } \\
\text { Beacon detection again }\end{array}$ \\
\hline Payment & $\begin{array}{l}\text { Walking; } \\
\text { Leaving }\end{array}$ \\
\hline
\end{tabular}




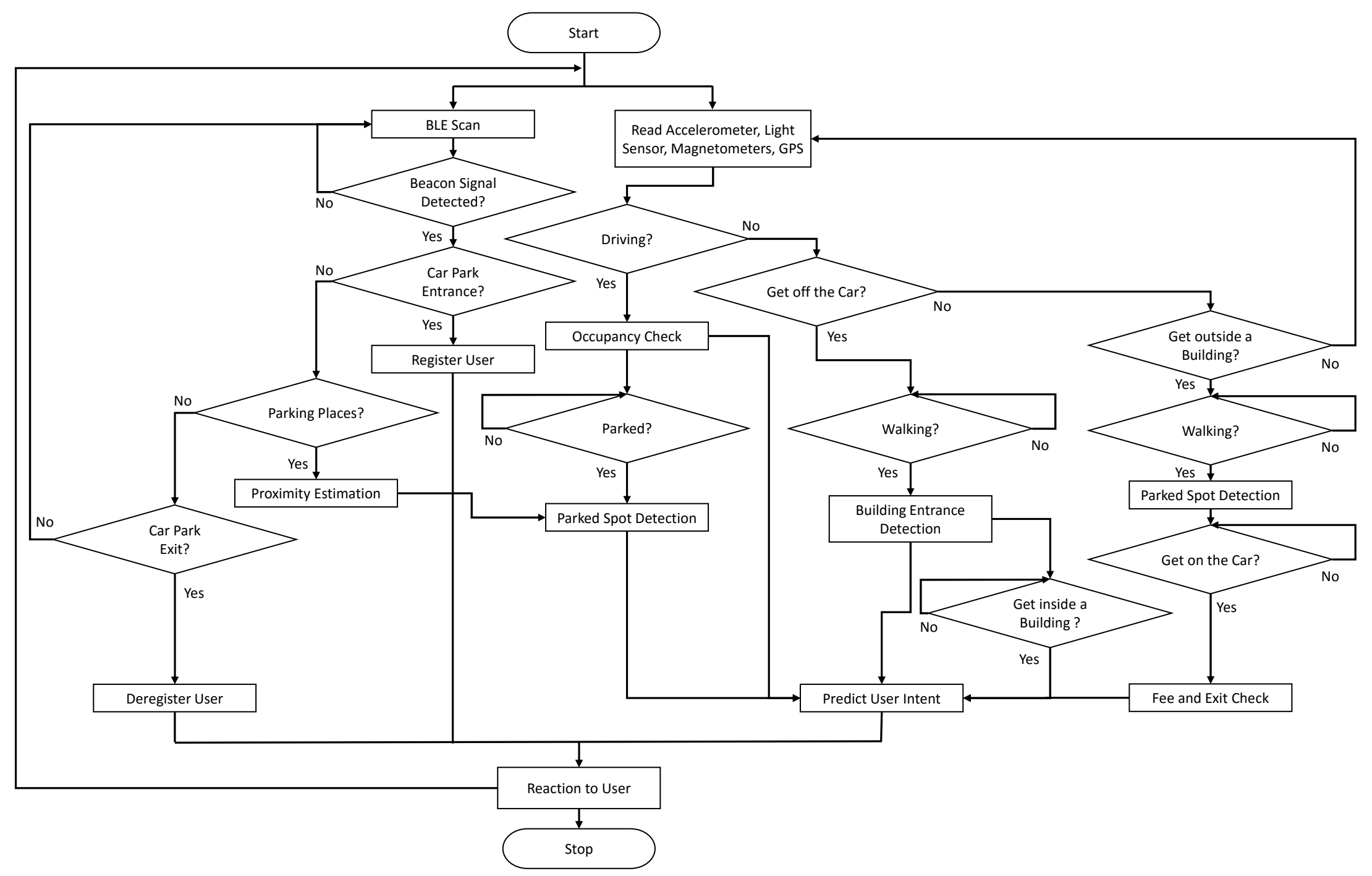

Figure 8. D-PARK operation flow chart. 


\section{User Service Experience Experiment}

This section shows the proof-of-concept prototype to implement and evaluate the novel positioning mechanism and the new parking service paradigm. To implement $\mathrm{D}$ PARK, this paper takes into account a smart parking lot on the campus with eleven BLE beacons running the iBeacon protocol. Figure 1 illustrates the testbed on the campus parking lot nearby a building. In terms of a cloud system, an apache, a MySQL, and a tomcat were set up on a desktop. The cloud resources are utilized for content delivery by BLE beacon IDs and service management. A mobile appliation running Android 6.0 was developed. For people sensing, the patterns and histories of BLE beacon and GPS signal reception and the sensing from sensors on the phone such as accelerometer and magnetic were analyzed. The experiments were fulfilled for positioning accuracy of our high-resolution proximity estimation mechanism and the level of user experience for total experience time and complication of using the system in parking situations. Table 3 details the system environments.

Table 3. Experimental environments.

\begin{tabular}{cc}
\hline Mobile OS & Android 8.0 \\
\hline Edge OS & Ubuntu 16.04 LTS \\
\hline Number of beacons & 3 \\
\hline BLE beacon broadcast interval & $100 \mathrm{~ms}$ \\
\hline Number of parking spot & $\begin{array}{c}\text { 8 for parking spots, } \\
\text { 2 for parking in the areas with no lines }\end{array}$ \\
\hline Number of collected data (per parking spot) & 6000 \\
\hline
\end{tabular}

Figure 9 illustrates the average service utilization time for the parking lot service to determine the service smartness. The legacy method means that combined service app utilization in the parking lot is based on existing smartphone apps to search the parking lot and the entrance of the building on the map and a manual parking place search by walking around the parking lot, while the developed app that relys on the proposed framework is solely exploited for the parking service. The legacy approach shows a much longer time period of 6.8 times ( $230 \mathrm{~s}$ ) than the proposed method ( $34 \mathrm{~s})$. Hence, according to this total utilization time for the entireity of the parking service procedures, users should experience much faster and dedicated service provisioning.

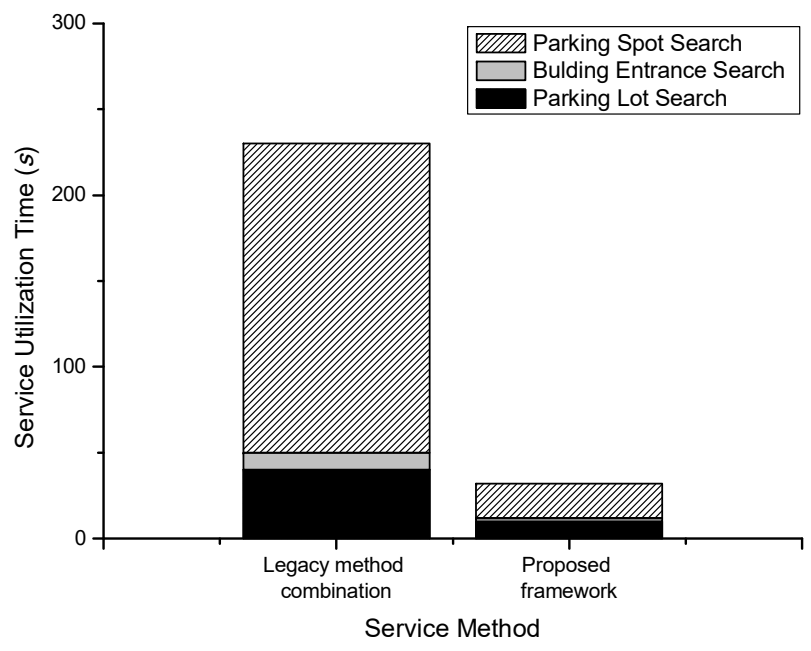

Figure 9. Service utilization time. 


\section{Conclusions and Future Work}

This paper has proposed a user-dedicated smart parking scheme with high-resolution proximity estimation, user/vehicle status sensing and user-intent-based smart service. In this scheme, the first study of BLE-beacon-based outdoor localization with a parking spot scale is reported. In addition, the data from sensors and wireless signals from smart mobile devices have been exploited to provide a comprehensive user and vehicle status sensing mechanism. Based on the sensing mechanism, the cloud-smart mobile device interaction was also implemented for intent-based smart service delivery to users in parking lots. The proof-of-concept shows implementation of this strategic system and the experimental results prove the system improves the performance of parking service operations.

In future work, we will add dynamic occupancy ratio analytics to improve user experience regarding finding the right parking spot at the right time. Furthermore, social computing will be adopted on the cloud system for achieving better user preference understanding for more accurate user intent detection. In addition, all technologies will be experienced by practical users for real surveys of their experiences with fine-grained parking service utilization points.

Funding: This research was supported by Basic Science Research Program through the National Research Foundation of Korea (NRF) funded by the Ministry of Education (2019R1I1A3A01062944).

Conflicts of Interest: The author declares no conflict of interest.

\section{References}

1. Palattella, M.R.; Dohler, M.; Grieco, A.; Rizzo, G.; Torsner, J.; Engel, T.; Ladid, L. Internet of Things in the 5G Era: Enablers, Architecture, and Business Models. IEEE J. Sel. Areas Commun. 2016, 34, 510-527. [CrossRef]

2. Lu, R.; Lin, X.; Zhu, H.; Shen, X. SPARK: A New VANET-Based Smart Parking Scheme for Large Parking Lots. In Proceedings of the IEEE INFOCOM 2009, Rio de Janeiro, Brazil, 19-25 April 2009.

3. Geng, Y.; Cassandras, C.G. New "Smart Parking" System Based on Resource Allocation and Reservations. IEEE Trans. Intell. Transp. Syst. 2013, 14, 1129-1139. [CrossRef]

4. Kotb, A.O.; Shen, Y.-C.; Zhu, X.; Huang, Y. iParker-A New Smart Car-Parking System Based on Dynamic Resource Allocation and Pricing. IEEE Trans. Intell. Transp. Syst. 2016, 17, 2637-2647. [CrossRef]

5. Tang, C.; Wei, X.; Zhu, C.; Chen, W.; Rodrigues, J.J.P.C. Towards Smart Parking Based on Fog Computing. IEEE Access 2018, 6, 70172-70185. [CrossRef]

6. Roman, C.; Liao, R.; Ball, P.; Ou, S.; Heaver, M. Detecting On-Street Parking Spaces in Smart Cities: Performance Evaluation of Fixed and Mobile Sensing Systems. IEEE Trans. Intell. Transp. Syst. 2016, 19, 2234-2245. [CrossRef]

7. Do, H.; Kim, J.; Lee, K.; Kim, D.; Chae, K.; Choi, J.Y. Implementation of CNN-Based Parking Slot Type Classification using Around View Images. In Proceedings of the 2020 IEEE International Conference on Consumer Electronics (ICCE), Las Vegas, NV, USA, 4-6 January 2020.

8. Lytras, M.D.; Visvizi, A. Who Uses Smart City Services and What to Make of It: Toward Interdisciplinary Smart Cities Research. Sustainability 2018, 10, 1998. [CrossRef]

9. Visvizi, A.; Lytras, M. (Eds.) Smart Cities: Issues and Challenges: Mapping Political, Social and Economic Risks and Threats; Elsevier: Amsterdam, The Netherlands, 2019.

10. Lee, C.; Park, S.; Yang, T.; Lee, S. Smart Parking with Fine-Grained Localization and User Status Sensing Based on Edge Computing. In Proceedings of the 2019 IEEE 90th Vehicular Technology Conference (VTC2019-Fall), Honolulu, HI, USA, 22-25 September 2019.

11. Sun, G.; Chen, J.; Guo, W.; Liu, K.J.R. Signal processing techniques in network-aided positioning: a survey of state-of-the-art positioning designs. IEEE Signal Process. Mag. 2005, 22, 12-23.

12. Faragher, R.; Harle, R. SmartSLAM. An efficient smartphone indoor positioning system exploiting machine learning and opportunistic sensing. In Proceedings of the 26th International Technical Meeting of the Satellite Division of The Institute of Navigation (ION GNSS+ 2013), Nashville, TN, USA, 16-20 September 2013; pp. 1006-1019.

13. Ferris, B.; Fox, D.; Lawrence, N. WiFi-SLAM using Gaussian process latent variable models. In Proceedings of the 20th International Joint Conference on Artificial Intelligence, Hyderabad, India, 6-12 January 2007; pp. 2480-2485.

14. Faragher, R.; Harle, R. Location Fingerprinting with Bluetooth Low Energy Beacons. IEEE J. Sel. Areas Commun. 2015, 33, 2418-2428. [CrossRef]

15. Zheng, V.W.; Zheng, Y.; Xie, X.; Yang, Q. Collaborative location and activity recommendations with GPS history data. In Proceedings of the The 19th International Conference on World Wide Web (WWW'10), Raleigh, NC, USA, 26-30 April 2010; pp. 1029-1038. 
16. Collotta, M.; Pau, G. A Novel Energy Management Approach for Smart Homes Using Bluetooth Low Energy. IEEE J. Sel. Areas Commun. 2015, 33, 2988-2996. [CrossRef]

17. Saguna, S.; Zaslavsky, A.; Chakraborty, D. Complex activity recognition using context-driven activity theory and activity signatures. ACM Trans. Comput. Hum. Interact. 2013, 20, 32. [CrossRef]

18. Vigneshwaran, S.; Sen, S.; Misra, A.; Chakraborti, S.; Balan, R.K. Using Infrastructure-provided Context Filters for Efficient Fine-grained Activity Sensing. In Proceedings of the 2015 IEEE International Conference on Pervasive Computing and Communications (PerCom), St. Louis, MO, USA, 23-27 March 2015.

19. Fan, Y.; Xie, L.; Yin, Y.; Lu, S. A Context Aware Energy-Saving Scheme for Smart Camera Phones Based on Activity Sensing In Proceedings of the 2015 IEEE 12th International Conference on Mobile Ad Hoc and Sensor Systems, Dallas, TX, USA, 19-22 October 2015; pp. 64-72.

20. She, J.; Crowcroft, J.; Fu, H.; Ho, P.-H. Smart Signage: A Draggable Cyber-physical Broadcast/Multicast Media System. IEEE Trans. Emerg. Top. Comput. 2013, 1, 232-243. [CrossRef]

21. He, Z.; Cui, B.; Zhou, W.; Yokoi, S. A Proposal of Interaction System between Visitor and Collection in Museum Hall by iBeacon. In Proceedings of the 2015 10th International Conference on Computer Science \& Education (ICCSE), Cambridge, UK, 22-24 July 2015; pp. 427-430.

22. Goodfellow, I.; Bengio, Y.; Courville, A. Deep Learning; MIT Press: Cambridge, MA, USA, 2016; ISBN 0262035618. 